Supporting Information

\title{
Effects of Ge Alloying on Device Characteristics of Kesterite-Based CZTSSe Thin Film Solar Cells
}

Dhruba B. Khadka, SeongYeon Kim, JunHo Kim*

Department of Physics, Incheon National University, 12-1 Songdo-dong Yeonsu-gu, 406-772

Incheon, South Korea

* Corresponding Author: JunHo Kim

E-mail: jhk@inu.ac.kr

Phone: $+82-32-835-8221$ 


\section{Tables and Figures}

Table S1. Elemental composition of CZTSSe and $\operatorname{CZTSSe}(\mathrm{Ge}-\mathrm{x})$ thin films and stoichiometric evaluation. Here $\mathrm{M}=\mathrm{Cu}+\mathrm{Zn}+\mathrm{Sn}+\mathrm{Ge}, \mathrm{N}=\mathrm{Zn}+\mathrm{Sn}+\mathrm{Ge}$, and $\mathrm{P}=\mathrm{Sn}+\mathrm{Ge}$. The errors of composition data given by EDS results are below $\pm 5 \%$ of measured values.

Table S2. Best device parameters of CZTSSe and CZGTSSe(Ge-x) thin film solar cells.

Table S3. Summary of average (Av.) and standard deviation (Sd.) of main device parameters of the respective devices as listed in Table S2 with the average of 6 representative solar cell devices.

Figure S1. The characteristics peaks trends of the XRD of 112 peaks and Raman of characteristic dominant peaks of A mode of CZTSSe and CZTSSe(Ge-3) thin films.

Figure S2. Cross sectional image of solar cell devices consisting of $\mathrm{SLG} / \mathrm{Mo} / \mathrm{MoSe}_{2} / \mathrm{CZTSSe}(\mathrm{Ge}-\mathrm{x}) / \mathrm{In}_{2} \mathrm{~S}_{3} / \mathrm{ZnO} / \mathrm{ITO}$ structure. Here, solar cell with absorber layer CZTSSe (a), and CZTSSe (Ge-x), where $\mathrm{x}=1,2,3,4$ given by b), (c), (d), and (e), respectively.

Figure S3. The plot of $[E \times \ln (1-E Q E)]^{2}$ vs $E=h v(e V)$ for the estimation of the band gap of corresponding absorber layers.

Figure S4. J-V curves of CZTSSe and CZTSSe(Ge-x) thin film solar cells devices. The device parameters are respective cells are given in Table S2.

Figure S5. J-V analysis of $\operatorname{CZTSSe}(\mathrm{Ge}-3)(\mathrm{a}, \mathrm{b})$ and $\mathrm{CZTSSe}(\mathrm{c}, \mathrm{d})$ solar cell devices adopting the one-diode model. ${ }^{1,2}$

Figure S6. Temperature dependent J-V characteristics (J-V-T) of CZTSSe and CZTSSe(Ge-3) solar cell devices.

Figure S7. Mott-Schottky plot and estimation of built-in-potential $\left(\mathrm{V}_{\mathrm{bi}}\right)$ of respective solar cell devices as described in reports ${ }^{2,3}$

Figure S8. Admittance spectra scan (C-f-T spectra) of CZTSSe and CZTSSe(Ge-3) solar cell devices. 
Table S1. Elemental composition of CZTSSe and $\operatorname{CZTSSe}(\mathrm{Ge}-\mathrm{x})$ thin films and stoichiometric evaluation. Here $\mathrm{M}=\mathrm{Cu}+\mathrm{Zn}+\mathrm{Sn}+\mathrm{Ge}, \mathrm{N}=\mathrm{Zn}+\mathrm{Sn}+\mathrm{Ge}$, and $\mathrm{P}=\mathrm{Sn}+\mathrm{Ge}$. The errors

\begin{tabular}{|c|c|c|c|c|c|c|c|c|c|c|c|c|c|}
\hline $\begin{array}{c}\text { Absorber } \\
\text { layer }\end{array}$ & $\begin{array}{c}\text { Expected } \\
\text { Ge/P } \\
(\%)\end{array}$ & $\begin{array}{l}\mathrm{Cu} \\
(\%)\end{array}$ & $\begin{array}{l}\mathrm{Zn} \\
(\%)\end{array}$ & $\begin{array}{l}\mathrm{Sn} \\
(\%)\end{array}$ & $\begin{array}{l}\mathrm{Ge} \\
(\%)\end{array}$ & $\begin{array}{l}\mathrm{Se} \\
(\%)\end{array}$ & $\begin{array}{c}\mathrm{S} \\
(\%)\end{array}$ & $\mathrm{Cu} / \mathrm{N}$ & $\mathrm{Zn} / \mathrm{P}$ & $\mathrm{Ge} / \mathrm{P}$ & $\mathrm{T} / \mathrm{P}$ & $\mathrm{S} / \mathrm{M}$ & $\mathrm{Se} / \mathrm{M}$ \\
\hline $\begin{array}{c}\text { CZTSSe } \\
(\mathrm{Ge}-0)\end{array}$ & 0 & 21.73 & 14.27 & 11.73 & 0 & 46.27 & 5.32 & 0.8367 & 1.2196 & 0 & 1 & 0.1115 & 0.9702 \\
\hline $\begin{array}{c}\text { CZGTSe } \\
(\mathrm{Ge}-1)\end{array}$ & $6 \pm 1$ & 22.61 & 14.66 & 11.52 & 0.51 & 46.74 & 5.75 & 0.8471 & 1.2186 & 0.04239 & 0.9576 & 0.1166 & 0.9481 \\
\hline $\begin{array}{c}\text { CZTSSe } \\
(\mathrm{Ge}-3)\end{array}$ & $16 \pm 2$ & 21.42 & 14.29 & 10.88 & 1.12 & 46.22 & 5.15 & 0.8139 & 1.1908 & 0.09333 & 0.9067 & 0.1079 & 0.9692 \\
\hline $\begin{array}{c}\text { CZTSSe } \\
(\mathrm{Ge}-4)\end{array}$ & $25 \pm 4$ & 22.51 & 15.24 & 10.68 & 2.15 & 49.42 & 3.53 & 0.8019 & 1.1878 & 0.1676 & 0.8324 & 0.06979 & 0.9771 \\
\hline
\end{tabular}

of stoichiometric data given by EDS results are below $\pm 5 \%$ of measured values. 

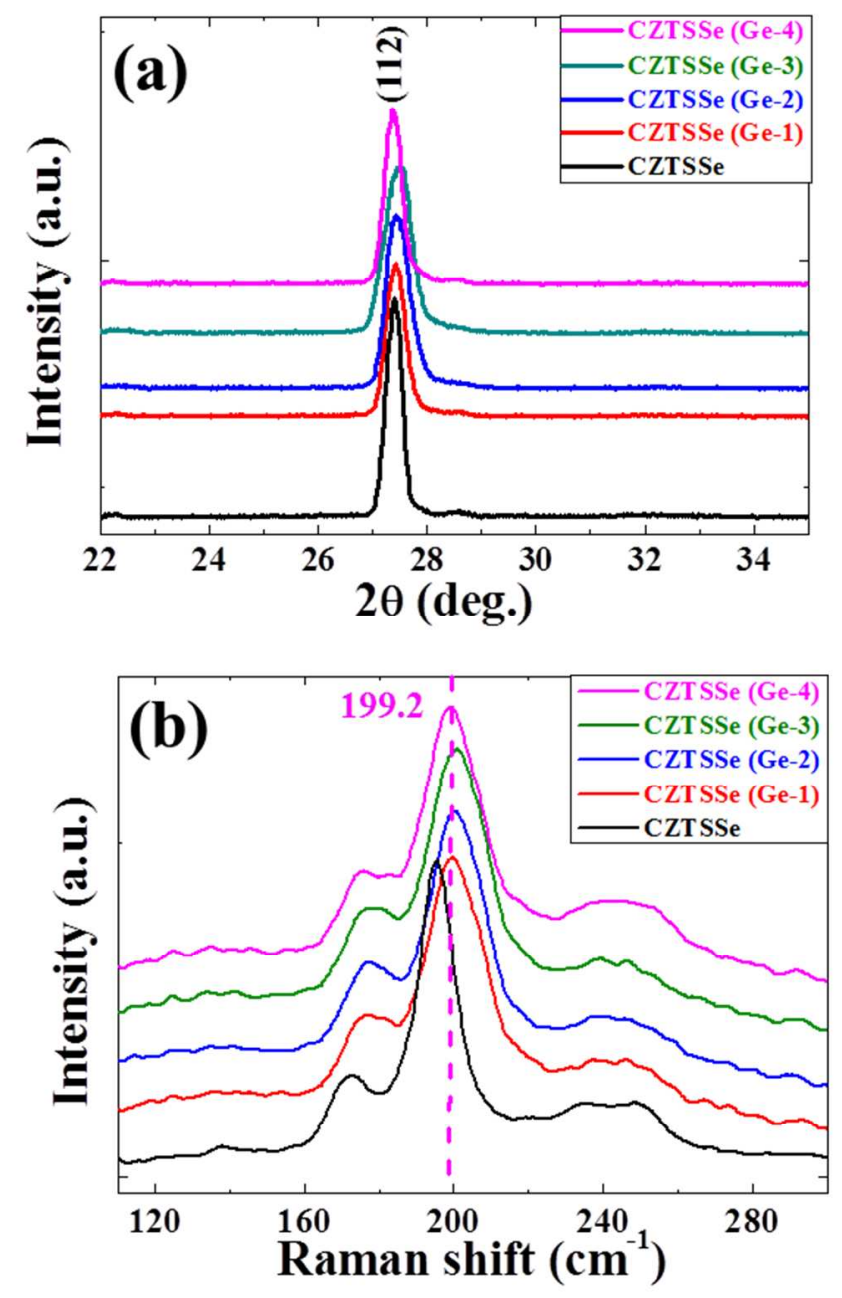

Figure S1. The characteristics peaks trends of the XRD of 112 peaks and Raman of characteristic dominant peaks of A mode of CZTSSe and CZTSSe(Ge-x) thin films. 

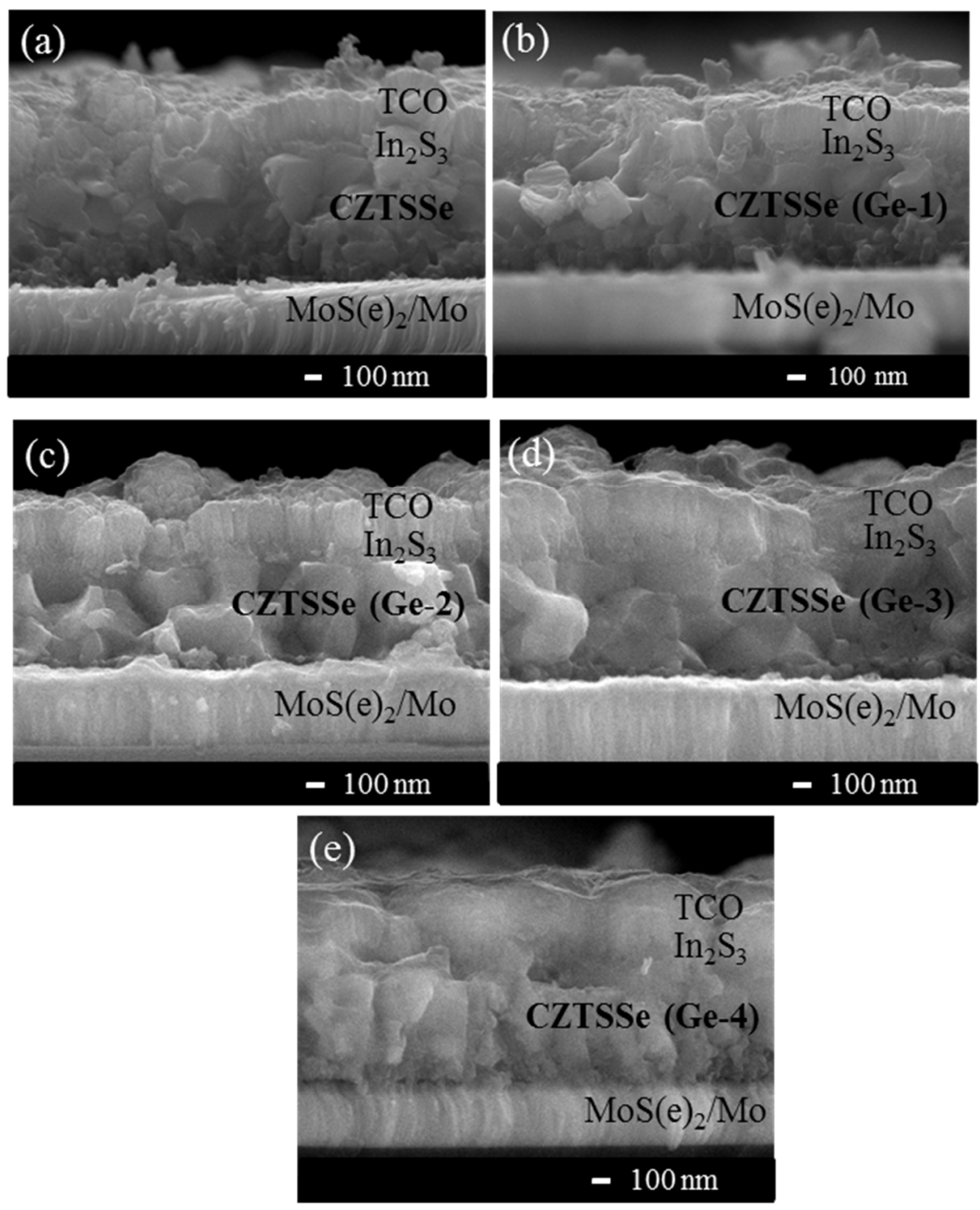

Figure S2. Cross sectional image of solar cell devices consisting of $\mathrm{SLG} / \mathrm{Mo} / \mathrm{MoSe}_{2} / \mathrm{CZTSSe}(\mathrm{Ge}-\mathrm{x}) / \mathrm{In}_{2} \mathrm{~S}_{3} / \mathrm{ZnO} / \mathrm{ITO}$ structure. Here, solar cell with absorber layer CZTSSe (a), and CZTSSe (Ge-x), where $\mathrm{x}=1,2,3,4$ given by b), (c), (d), and (e), respectively. 


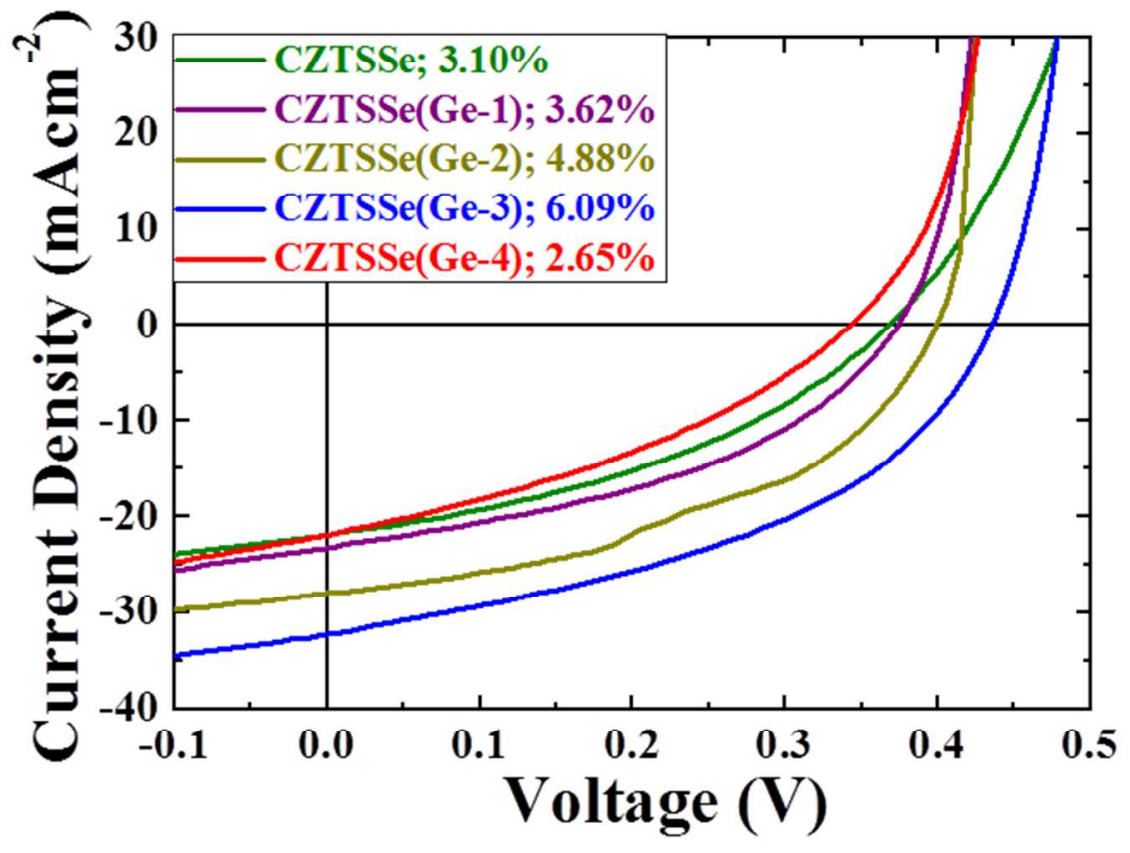

Figure S3. J-V curves of CZTSSe and CZTSSe(Ge-x) thin film solar cells devices. The device parameters are respective cells are given in Table S2. 
Table S2. Best device parameters of CZTSSe and CZTSSe(Ge-x) thin film solar cells

\begin{tabular}{|c|c|c|c|c|c|}
\hline Device parameters & CZTSSe & CZTSSe(Ge-1) & CZTSSe(Ge-2) & CZTSSe(Ge-3) & CZTSSe(Ge-4) \\
\hline Voc $(\mathrm{V})$ & 0.3681 & 0.3736 & 0.3991 & 0.4351 & 0.3426 \\
\hline $\mathrm{J}_{\mathrm{SC}}\left(\mathrm{mAcm}^{-2}\right)$ & 21.92 & 23.23 & 28.05 & 32.31 & 21.91 \\
\hline $\mathrm{FF}(\%)$ & 38.47 & 41.79 & 43.65 & 43.38 & 35.27 \\
\hline$\eta(\%)$ & 3.104 & 3.624 & 4.901 & 6.098 & 2.647 \\
\hline $\mathrm{R}_{\mathrm{SH}}(\Omega)$ & 622.926 & 579.384 & 463.657 & 619.509 & 331.855 \\
\hline $\mathrm{R}_{\mathrm{S}}(\Omega)$ & 168.791 & 94.346 & 50.624 & 64.526 & 102.547 \\
\hline $\mathrm{E}_{\mathrm{g}} \pm 0.01 \mathrm{eV}$ & 1.118 & 1.120 & 1.124 & 1.132 & 1.130 \\
\hline
\end{tabular}

Table S3. Summary of average (Av.) and standard deviation (Sd.) of main device parameters of the respective devices as listed in Table S2 with the average of 6 representative solar cell devices.

\begin{tabular}{|c|c|c|c|c|c|c|c|c|}
\hline Device parameters & $\begin{array}{c}\text { Av. Voc } \\
(\mathrm{V})\end{array}$ & $\begin{array}{c}\text { Sd. Voc } \\
(\mathrm{V})\end{array}$ & $\begin{array}{c}\text { Av. } \mathrm{J}_{\mathrm{SC}} \\
\left(\mathrm{mAcm}^{-2}\right)\end{array}$ & $\begin{array}{c}\text { Sd. } \mathrm{J}_{\mathrm{SC}} \\
\left(\mathrm{mAcm}^{-2}\right)\end{array}$ & $\begin{array}{c}\text { Av. FF } \\
(\%)\end{array}$ & $\begin{array}{c}\text { Sd. FF } \\
(\%)\end{array}$ & $\begin{array}{c}\text { Av. } \eta \\
(\%)\end{array}$ & $\begin{array}{c}\text { Sd. } \eta \\
(\%)\end{array}$ \\
\hline CZTSSe & 0.3432 & 0.0118 & 19.82 & 1.21 & 36.58 & 1.82 & 2.758 & 0.36 \\
\hline CZTSSe(Ge-1) & 0.3533 & 0.0116 & 21.38 & 1.08 & 39.27 & 1.67 & 3.268 & 0.78 \\
\hline CZTSSe(Ge-2) & 0.3792 & 0.0112 & 26.85 & 0.95 & 41.35 & 0.86 & 4.425 & 0.534 \\
\hline CZTSSe(Ge-3) & 0.4154 & 0.0095 & 31.08 & 0.67 & 42.94 & 0.58 & 5.856 & 0.287 \\
\hline CZTSSe(Ge-4) & 0.3026 & 0.0114 & 18.74 & 1.68 & 31.78 & 2.36 & 1.72 & 0.875 \\
\hline
\end{tabular}



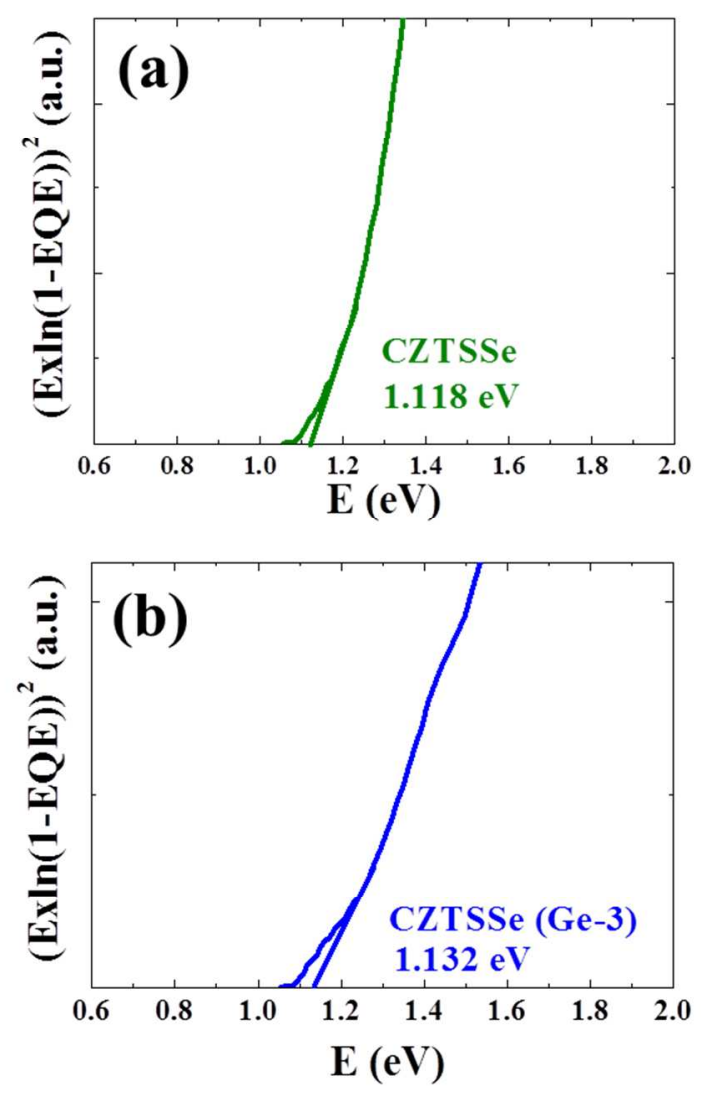

Figure S4. The plot of $[E \times \ln (1-E Q E)]^{2}$ vs $E=h v(e V)$ for the estimation of the band gap of corresponding absorber layers. 
To deal with the limiting electrical factors of the devices, a detailed analysis of J-V curves was carried out using the one-diode equation given $b y^{24}$,

$$
J(V)=J_{o}\left[\exp \left(\frac{q\left(V-R_{S} J\right)}{A k_{B} T}\right)\right]+\frac{V-R_{S} J}{R_{S h}}-J_{L}(V)
$$

Where $J_{o}$ is saturation current density, A is diode ideality factor, $J_{L}$ is photocurrent density and $\mathrm{R}_{\mathrm{S}}$ and $\mathrm{R}_{\mathrm{Sh}}$ are the series and shunt resistance.
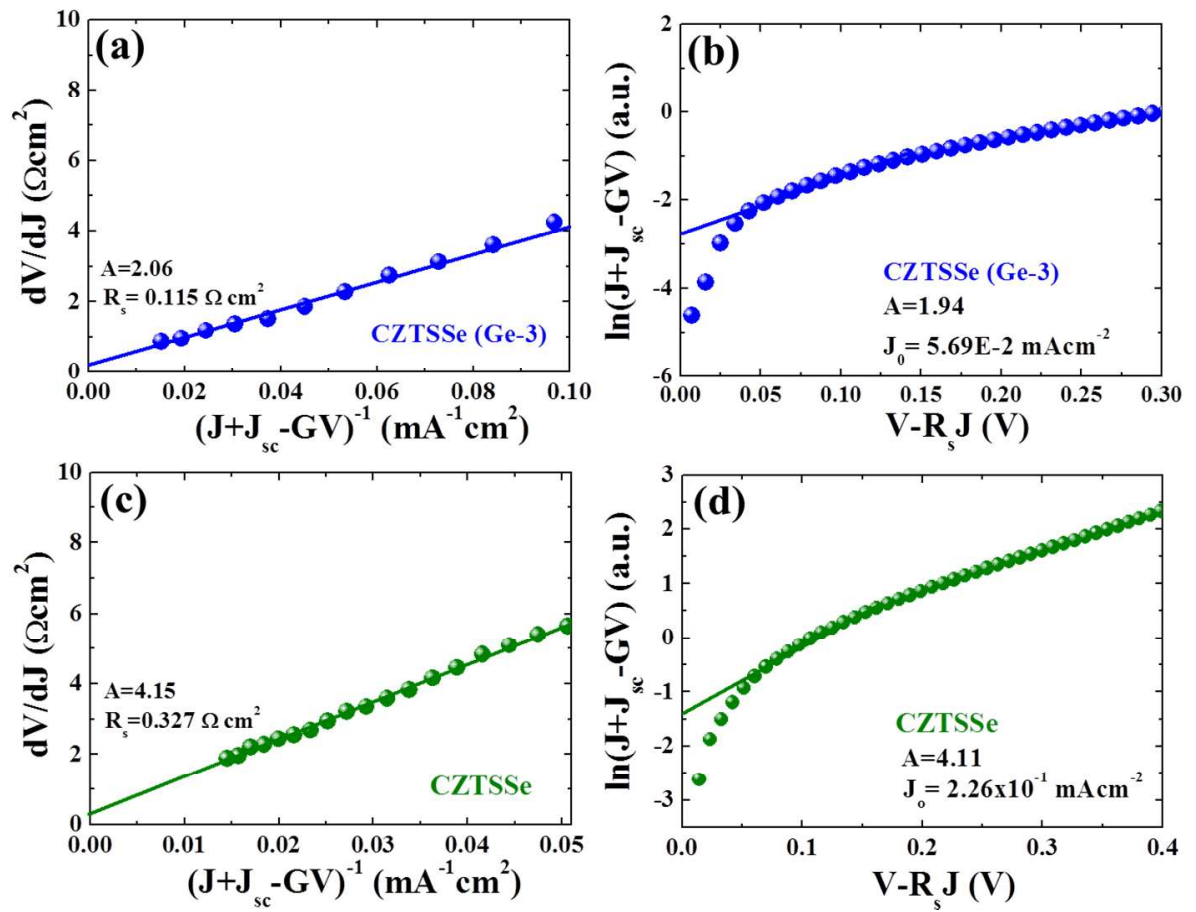

Figure S5. J-V analysis of CZTSSe(Ge-3) $(\mathrm{a}, \mathrm{b})$ and CZTSSe $(\mathrm{c}, \mathrm{d})$ solar cell devices adopting the one-diode model. ${ }^{1,2}$ 

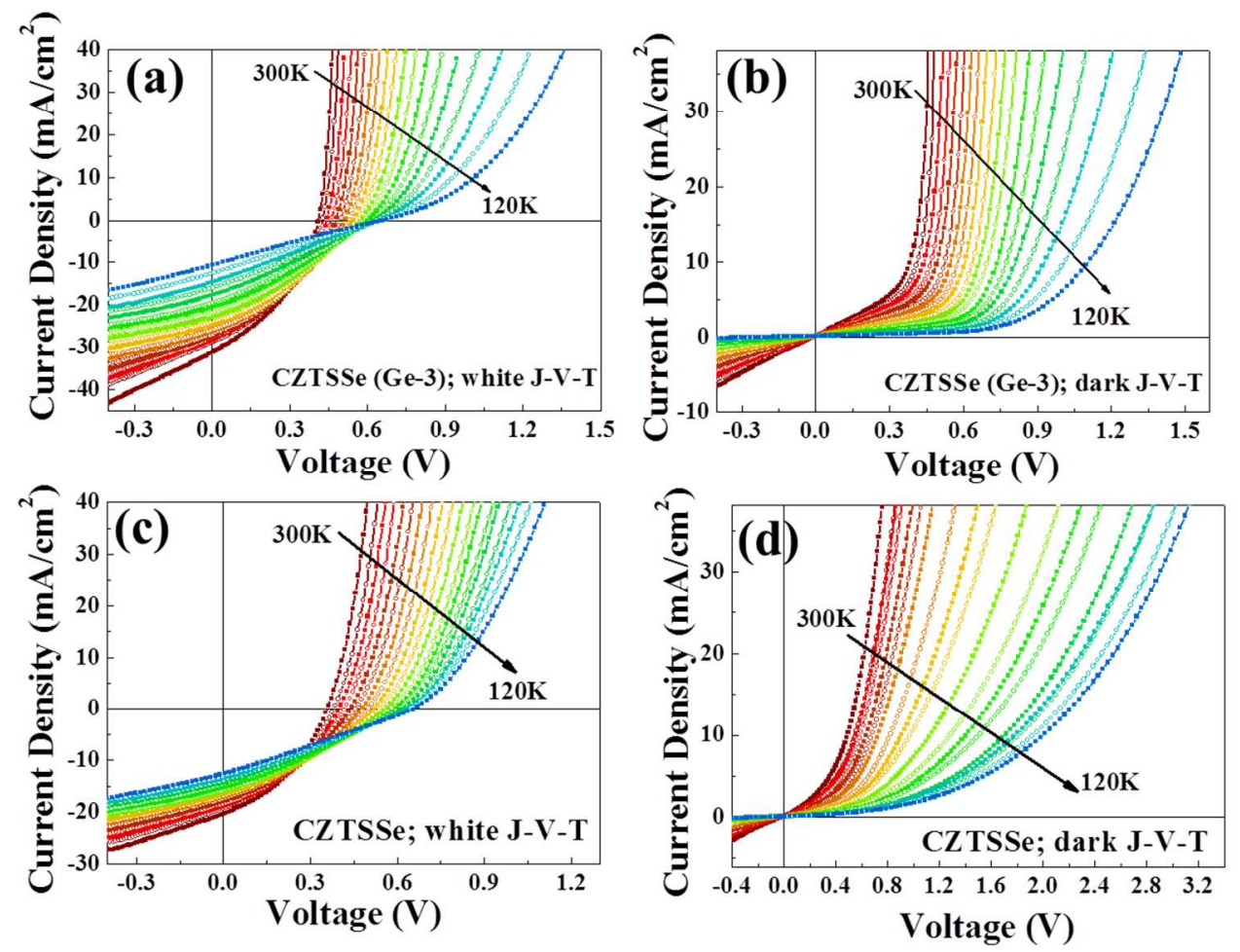

Figure S6. Temperature dependent J-V characteristics (J-V-T) of CZTSSe and CZTSSe(Ge-3) solar cell devices. 
The capacitance voltage $(\mathrm{C}-\mathrm{V})$ profiles of devices were measured to estimate charge carrier density profile and built in potential at the junction by Mott-Schottkey plot given by following relation, ${ }^{2,3}$

$$
\begin{gathered}
\frac{1}{C^{2}}=\frac{2}{q \epsilon \epsilon_{0} A^{2} N_{A}}\left(V_{b i}-V_{d c}\right) \\
\mathrm{N}_{A}^{C V}(x)=-\frac{2}{q \epsilon \epsilon_{0} A^{2}}\left[\frac{d\left(C^{-2}\right)}{d V_{d c}}\right]^{-1}=-\frac{C^{3}}{q \epsilon \epsilon_{0} A^{2}}\left[\frac{d C}{d V_{d c}}\right]^{-1}
\end{gathered}
$$

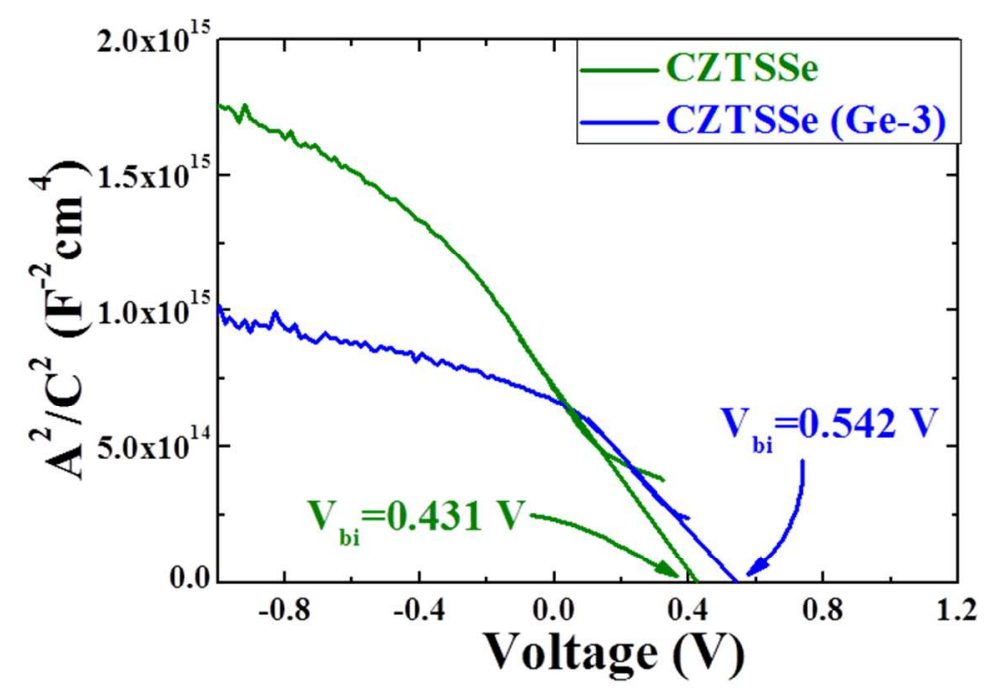

Figure S7. Mott-Schottky plot and estimation of built-in-potential $\left(\mathrm{V}_{\mathrm{bi}}\right)$ of respective solar cell device. 

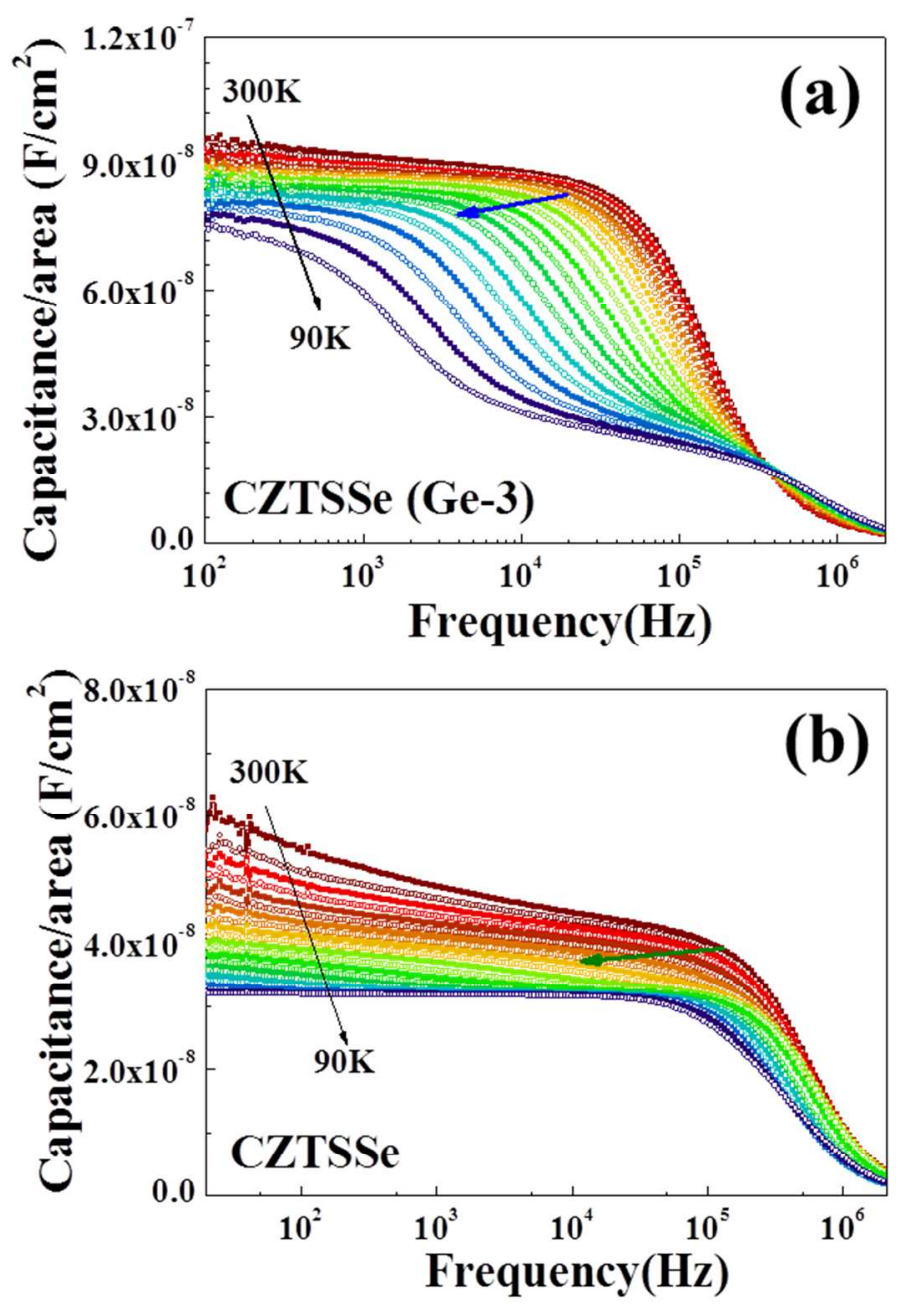

Figure S8. Admittance spectra scan (C-f-T spectra) of CZTSSe and CZTSSe(Ge-3) solar cell devices. 


\section{REFRERENCES}

[1] Hegedus, S. S.; Shafarman, W. N. Thin-Film Solar Cells: Device Measurements and Analysis. Prog. Photovoltaics: Res. Appl. 2004, 12, 155-176.

[2] Abou-Ras, D.; Kirchartz, T.; Rau, U. Advance Characterization Techniques for Thin Film Solar cell. Wiley-VCH Verlag GmbH \& Co. KGaA, Weinheim, Germany 2011, $81-103$

[3] Heath, J. T.; Cohen, J. D.; Shafarman, W. N. Bulk and Metastable Defects in $\mathrm{CuIn}_{1-\mathrm{x}} \mathrm{Ga}_{\mathrm{x}} \mathrm{Se}_{2}$ Thin Films Using Drive-Level Capacitance Profiling. J. Appl. Phys. 2004, $95,100-110$. 